\title{
Clinical and Experimental Evaluation of Urinary Histidine Derivatives as an Index of Folic Acid Metabolism
}

\author{
III. Clinical Observation in Liver Disease
}

\author{
SHOJIRO ISHIHARA ${ }^{1}$ \\ The First Division, Department of Internal Medicine, Faculty \\ of Medicine, Kyoto University, Kyoto (Post No. 606)
}

(Received February 25, 1969)

\begin{abstract}
Urinary excretion of formiminoglutamic acid (FIGLU) and urocanic acid after a loading with $15 \mathrm{~g}$ of L-histidine was observed in 53 patients with various liver diseases. Of 53 patients $58.5 \%$ was in excess of FIGLU, $52.0 \%$ for urocanic acid, and $64.0 \%$ for the two combined derivatives. The frequent abnormality in these histidine derivatives in liver diseases suggested a widespread deficiency in folic acid metabolism in such patients.

For analyzing the mechanism of an excessive excretion of histidine derivatives, the therapeutic trials with minimal daily doses of folic acid, tetrahycrofolic acid (THF), and doses of coenzyme $B_{12}$ and $C N-B_{12}$ were carried out in seven patients with liver diseases and changes in the urinary histidine derivatives were observed.

Four out of seven patients responded to folic acid, and oneof two patients refractory to folic acid responded to THF.

It was of particular interest that a case in which both folic acid and THF were ineffective, responded to both coenzyme $B_{12}$ and vitamin $B_{12}$. The mechanism of an increased excretion of histidine derivatives in the liver disease was discussed.
\end{abstract}

The existence of megaloblastic change in patients with chronic liver disease suggested that liver damage had the possibility to alter the storage or the metabolism of folic acid in whose deficiency the morphologic abnormality was characteristic $(14,27)$. In earlier studies megaloblastic anemia was chosen as an index of folic acid deficiency, but its occurrence as a complication of liver disease was found less frequent. Jarrold and Vilter reviewed the marrows of 30 patients with liver cirrhosis and found three to be megaloblastic (14).

However, the subsequent studies of plasma

\footnotetext{
1 石原象二郎
}

clearance of folate activity and of serum folate activity have revealed more frequent appearance of folate deficiency in liver diseases. The rapid clearances sometimes observed in patients with inadequate nutritional intake (27) or malabsorption (9) in the absence of megaloblastic changes of the bone marrow would represent the evidence of subclinical folate deficiency. Klipstein and Lindenbaum have found that in 19 out of 55 patients with liver diseases serum folate concentrations were subnormal and folate clearances rapid (15).

Recently, the excretion of formimino- 
glutamic acid (FIGLU) and urocanic acid has been shown to be a reliable index of folate -deficiency $(18,21)$. FIGLU and urocanic acid are the intermediates in the pathway of histidine breakdown to glutamic acid, and in some stage of the metabolism, the active form of folic acid, tetrahydrofolic acid (THF), has been found to be necessary as a coenzyme $(25,26,28,31,32)$. A number of studies have reported that patients with a variety of clinical disorders showed abnormal excretion of FIGLU and urocanic acid in urine $(2,3,5,8$, $16,23)$, and the relative importance of the latter was recognized in our preceding report (13).

When there is any interference with folic acid coenzymes, it is naturally anticipated that these histidine derivatives would accumulate and be excreted in urine. At the same time, it would be supposed that the lowered activity of liver enzymes associated with the histidine metabolism causes depletion of THF and increases urinary excretion of histidine derivatives. Daft postulated that excessive excretion of FIGLU might result from impaired liver function (10), and it was confirmed by Carter et al. in a study of 30 alcoholic and cirrhotic patients, 29 had an excessive excretion of FIGLU after histidine loading (5).

In the present study, the histidine derivatives were chosen as an index for estimating the folic acid metabolism in liver diseases. First, the frequency of appearance of increased histidine derivatives in urine was observed and secondly, the response of the derivatives to the therapeutic trial with folic acid, vitamin $B_{12}$, and their active forms was serially observed.

\section{Materials AND Method}

\section{Patients Studied}

Eifty-three patients with various liver diseases were selected for this study. Fourteen of 53 patients were with acute hepatitis, 12 with chronic hepatitis, 20 with liver cirrhosis, and seven with liver cancer. The diagnosis was made by biopsy or autopsy material in 45 patients. In the remaining patients, the diagnosis was based on the liver function tests and physical findings. In addition. observations were made on 21 patients with various neoplastic diseaes. The patients in whom the diagnosis of malignancy was established were divided into two groups: those with and without liver dysfunction. The existence of liver impairment in the patients was decided on the basis of liver function tests as follows: icteric index more than six, bromsulfalein (BSP) retention more than $5 \%$ at 30 minutes, serum glutamic oxaloacetic transaminase (SGOT) more than 40 units, and cephalin flocculations more than four units. Using the tests as discriminants, ten of 21 patients with malignancy showed the existence of liver dysfunction and the remaining patients the absence.

Five patients with chronic hepatitis and two with liver cirrhosis were selected for serial obervations concerning therapeutic trial with folic acid, THF, coenzyme $\mathrm{B}_{12}$, and vitamin $\mathrm{B}_{12}{ }^{\circ}$

\section{Therapeutic Trials}

The dose of therapeutic material used was as little as $0.1 \mathrm{mg} /$ day of folic acid and THF for 10 days, and $0.1 \mu \mathrm{g} /$ day of coenzme $\mathrm{B}_{12}$ and $0.5 \mu \mathrm{g} /$ day of vitamin $B_{12}$ for five days. All of the compounds were administered intramuscularly. During the trials, the urinary histidine derivatives, liver function, and hematological changes were estimated.

All compounds were obtained commercially, and cyanocobalamin $\left(\mathrm{CN}-\mathrm{B}_{12}\right)$ was used as vitamin $\mathrm{B}_{12}$ and 5,6-dimethylbenzimidazolylcobamide (DBCC) as coenzyme $\mathrm{B}_{12}$.

3. The Method of Estimating Urinary Histidine Derivatives

Principally, the method used in this study was a modification of the method of Chanarin and Bennett (7). The details have been fully described in the preceding report (13). All the patients studied were given orally a dose of $15 \mathrm{~g}$ of L-histidine hydrochloride and 24-hour urine was collected thereafter. Prior to urine collections, the container was added with $5 \mathrm{ml}$ of $3 \mathrm{~N} \mathrm{HCl}$ to prevent the destruction of FIGLU. An aliquot was stored at $-20^{\circ}$ for the assay.

\section{RESULTS}

\section{Urinary Excretion of Histidine Deriva-} tives in Patients with Liver Diseases

The mean 24-hour excretion of FIGLU and urocanic acid, and their upper limit in normal subjects after loading of $15 \mathrm{~g}$ of $\mathrm{L}$ histidine have been estimated in the preceding report (13). The upper limit was $30 \mathrm{mg}$ for each histidine derivativeand $60 \mathrm{mg}$ for the combination of the two. 


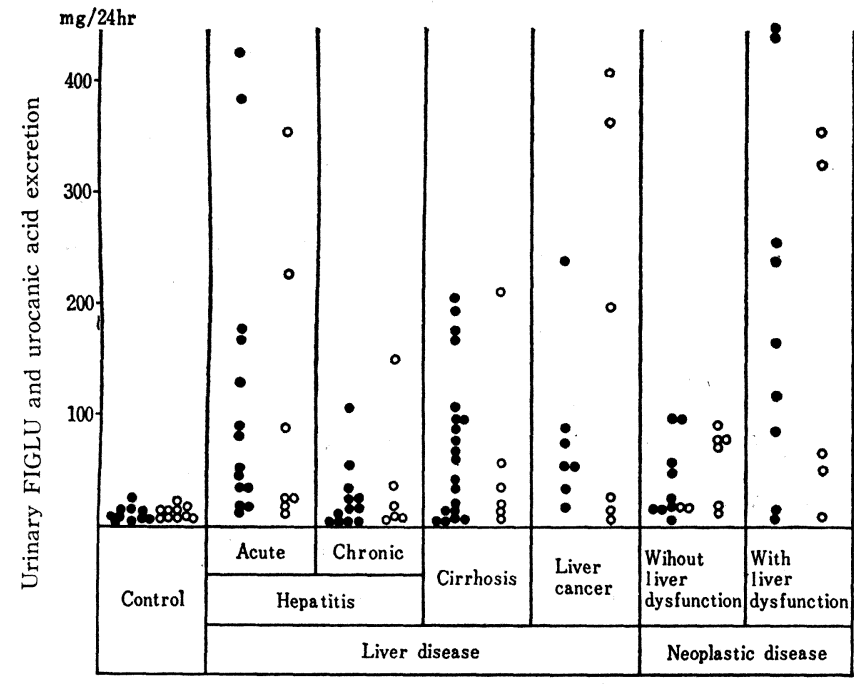

FIG. 1 Urinary FIGLU and urocanic acid excretion in patients with various diseases.

- FIGLU; and $O$, urocanic acid.
Table 1 summarizes the hematological findings and the amounts of FIGLU and urocanic acid excretion after histidine loading in 53 patients with various liver diseases. Similarly, Fig. 1 shows the tendency of the histidine derivative excretion in the same patients with liver diseases in association with 21 patients with neoplastic diseases. Patients with various neoplastic diseases were divided into two groups; namely, those with and without liver dysfunction for the purpose of observing the correlation between the abnormal amounts of the derivatives and liver impairement.

It can be seen in Table 1 that there was no firm, mutual relation between the amounts of histidine derivatives and hematological findings, i.e., red and white blood cell
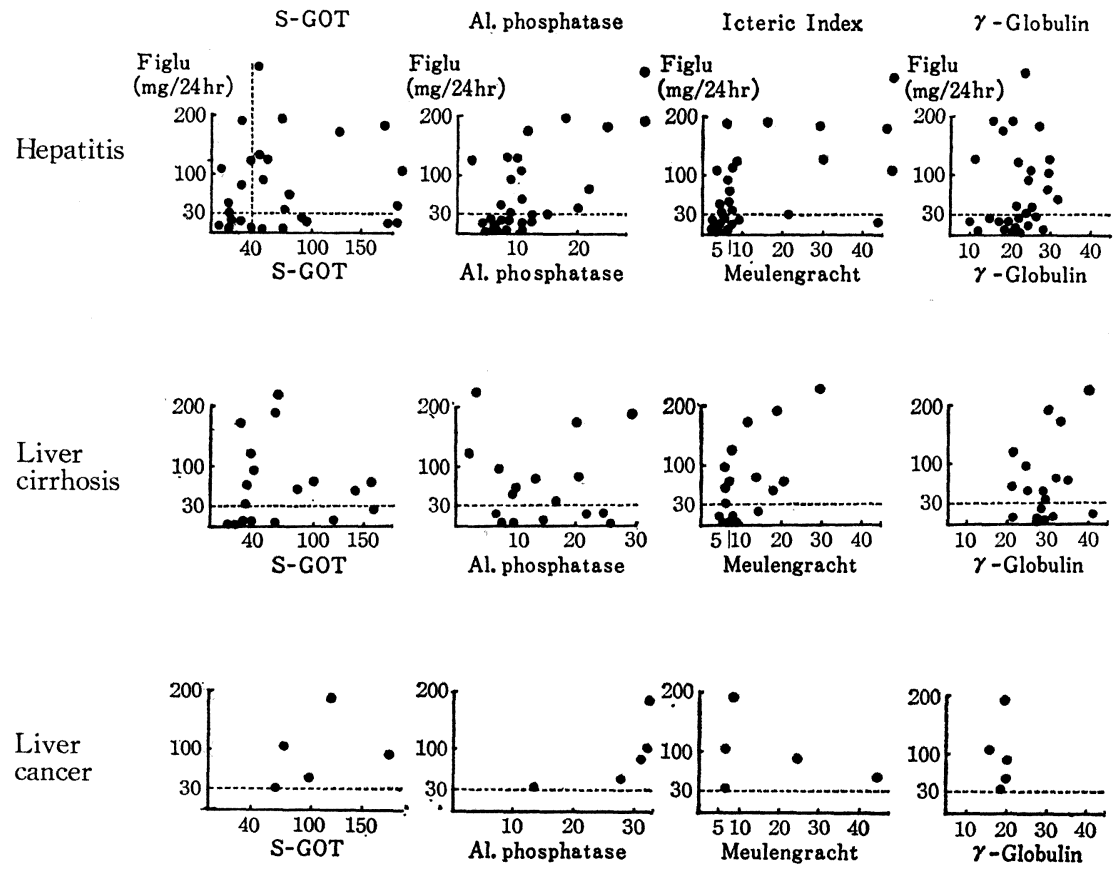

FIG. 2 Correlation between the amount of urinary FIGLU and each liver function tests in chronic and acute hepatitis, cirrhosis, and liver cancer. 
count, hematocrit, hemoglobin concentration and reticulocyte count.

It can also be seen in Table 1 and Fig. 1 that the excretion in xcesse of the two derivatives was found in 14 of 26 patients with acute and chronic hepatitis, in 11 of 20 patients with liver cirrhosis, and in six of seven patients with liver cancer the excretion of FIGLU; and in five of 13 patients with the hepatitis, in four of six with cirrhosis, and in four of six with liver cancer the excretion of urocanic acid. Of the total 53 patients with liver diseases, the frequency of abnormal excretion of the derivatives was $58.5 \%$ for FIGLU, $52.0 \%$ for urocanic acid, and $64.0 \%$ for the combined two derivatives. When this result was compared with those of the preceding report (13), the appearance of the

TABLE 1

Hematological findings and histidine derivatives in liver diseases

\begin{tabular}{|c|c|c|c|c|c|c|c|c|}
\hline \multirow{2}{*}{$\begin{array}{l}\text { No. of } \\
\text { case }\end{array}$} & \multirow{2}{*}{ Diagnosis } & \multicolumn{5}{|c|}{ Hematological findings } & \multicolumn{2}{|c|}{ Histidine derivatives } \\
\hline & & $\mathrm{RBC}$ & WBC & $\mathrm{Hb}$ & $\mathrm{Ht}$ & Reticulo. & FIGLU & $\begin{array}{l}\text { Urocanic } \\
\text { acid }\end{array}$ \\
\hline & & $\times 10^{6}$ & $\times 10^{3}$ & $g$ & per cent & per mil & \multicolumn{2}{|c|}{$m g / d a y$} \\
\hline 1 & Chronic hepatitis & 4.48 & 9.6 & 14.9 & & 10 & 8 & 6 \\
\hline 2 & ," & 2.95 & 5.4 & 11.4 & 32 & 17 & 53 & 19 \\
\hline 3 & ," & 2.64 & 3.5 & 9.0 & 28 & 22 & 24 & \\
\hline 4 & , & 4.15 & 7.9 & 14.0 & & & 14 & \\
\hline 5 & ," & 5.03 & 5.3 & 16.4 & 41 & 16 & 24 & \\
\hline 6 & ," & 4.24 & 7.9 & 13.5 & & 30 & 4 & \\
\hline 7 & , & 4.47 & 6.8 & 13.2 & 40 & 10 & 105 & 33 \\
\hline 8 & ," & 3.95 & 4.6 & 11.7 & 34 & & 0 & \\
\hline 9 & ," & 4.22 & 5.3 & 11.2 & 36 & 14 & 14 & 6 \\
\hline 10 & , & 5.01 & 5.0 & 14.5 & 44 & 18 & 2 & 4 \\
\hline 11 & , & 4.52 & 4.8 & 14.6 & 46 & & 32 & 147 \\
\hline 12 & , & & 3.7 & 11.7 & 35 & 11 & 4 & \\
\hline 13 & Acute hepatitis & 5.00 & 4.9 & 15.9 & & & 46 & \\
\hline 14 &, & 4.05 & 4.6 & 12.9 & 42 & & 23 & \\
\hline 15 & ", & 3.44 & 6.5 & 12.3 & 44 & 32 & 41 & \\
\hline 16 & ," & 3.68 & 6.4 & 11.1 & 31 & & 16 & \\
\hline 17 & ", & 3.54 & 6.7 & 11.8 & 38 & & 384 & \\
\hline 18 & ", & 3.15 & 6.9 & 10.2 & & 21 & 33 & 21 \\
\hline 19 & , & 3.69 & 6.5 & 11.5 & 39 & 40 & 88 & 225 \\
\hline 20 & ", & 2.94 & 4.6 & 10.7 & & 38 & 81 & 84 \\
\hline 21 & ," & 3.95 & 4.8 & 12.5 & 40 & & 176 & \\
\hline 22 & , & 3.87 & 5.5 & 12.7 & 37 & 13 & 167 & 20 \\
\hline 23 & ", & 3.18 & 4.9 & 10.5 & 34 & 14 & 129 & 26 \\
\hline 24 & ", & 4.16 & 8.9 & 13.8 & 40 & & 448 & 354 \\
\hline 25 & , & 3.19 & 3.4 & 10.2 & 31 & & 34 & \\
\hline 26 & ", & 5.04 & 6.3 & 10.1 & 46 & & 10 & 13 \\
\hline 27 & Liver cirrhosis & 3.37 & 4.8 & 7.4 & 29 & 14 & 204 & 209 \\
\hline 28 & , & 4.04 & 12.3 & 13.3 & 40 & 18 & 3 & 55 \\
\hline 29 & ," & 2.84 & 3.6 & 10.2 & 32 & & 64 & 5 \\
\hline 30 & ", & 4.33 & 4.1 & 14.8 & & 2 & 6 & \\
\hline 31 & , & 2.15 & 2.5 & 9.6 & & 29 & 171 & \\
\hline 32 & ", & 3.75 & 4.4 & 11.5 & 38 & & 96 & \\
\hline 33 & ," & 3.86 & 3.3 & 10.6 & 40 & & 71 & \\
\hline 34 & , & 3.42 & 1.1 & 10.6 & 33 & & 34 & \\
\hline 35 & ," & 3.23 & 3.0 & 8.1 & 31 & 22 & 191 & \\
\hline 36 & ", & 4.33 & 7.9 & 16.0 & 45 & 15 & 0 & \\
\hline 37 & , & 3.80 & 3.0 & 13.5 & 38 & 6 & 8 & \\
\hline 38 & ," & 4.33 & 4.7 & 14.5 & 45 & & 15 & 32 \\
\hline 39 & ", & 3.53 & 9.6 & 11.0 & 36 & 22 & 28 & \\
\hline 40 & , & 3.81 & 5.4 & 13.4 & 34 & 32 & 92 & \\
\hline 41 & ," & 3.63 & 1.0 & 10.5 & 28 & & 9 & \\
\hline 42 & ", & 3.64 & 2.0 & 12.5 & 35 & & 60 & 81 \\
\hline 43 & $"$ & & 4.2 & 12.0 & 34 & & 91 & \\
\hline 44 & $"$ & 2.78 & 8.5 & 7.9 & 32 & & 12 & \\
\hline 45 & $"$ & 1.84 & 7.7 & 4.5 & 19 & & 209 & \\
\hline 46 & $"$ & 3.76 & 6.5 & 11.4 & 37 & & 4 & 10 \\
\hline 47 to 53 & Liver cancer ${ }^{a}$ & & & & & & & \\
\hline
\end{tabular}

${ }^{a}$ See the previous report (13) 
abnormal derivatives was a little more frequent in liver diseases.

The mean value of the urinary derivatives in the patients with neoplastic diseases with liver dysfunction was $195 \mathrm{mg}$ of FIGLU and $158 \mathrm{mg}$ of urocanic acid, and the mean value of those without liver dysfunction was $37 \mathrm{mg}$ of FIGLU and $59 \mathrm{mg}$ of urocanic acid. The former significantly exceeded the latter. However, the result cannot surely support the correlation of the histidine derivatives to liver impairment for the reason that the cause of the excessive amounts of the derivatives was not clear whether the tumor was so extensive as to in clude the liver metastasis or only the liver impairment existed.

Fig. 2 shows the correlation between the amount of FIGLU and liver function tests in patients with liver diseases. For this purpose, SGOT, icteric index, alkaline phosphatase, and serum $\gamma$-globulin were chosen. As can be seen in Fig. 2, the degree of the abnormal FIGLU excreted in the urine had no direct correlation to each liver function test.

2. Therapeutic Trials with Folic Acid and $T H F$

In the next series of observations, the therapeutic trial with daily minimal dose of folic acid was applied to the randomly selected five patients with chronic hepatitis and two patients with liver cirrhosis (Case 1-7). Patients were given individually a daily dose of $0.1 \mathrm{mg}$ of folic acid for successive ten days, and the response of urinary histidine derivative excretion was estimated. The results are summarized in Fig. 3.

A marked decline of the derivatives to the normal range was found in four of seven cases on the fifth day of the course (Case $1,2,3$, and 5 ), and the excretion of the derivatives remained in the normal range in these four cases on the 10th day. However, in two of these cases (Case 3 and 5), the derivatives began to increase again when folic acid was stopped, reaching the abnormal range. The remaining three cases (Case 4,6, and 7) showed no response to the folic acid therapy throughout the period of observation.

Fig. 4 shows the effectof folic acid on

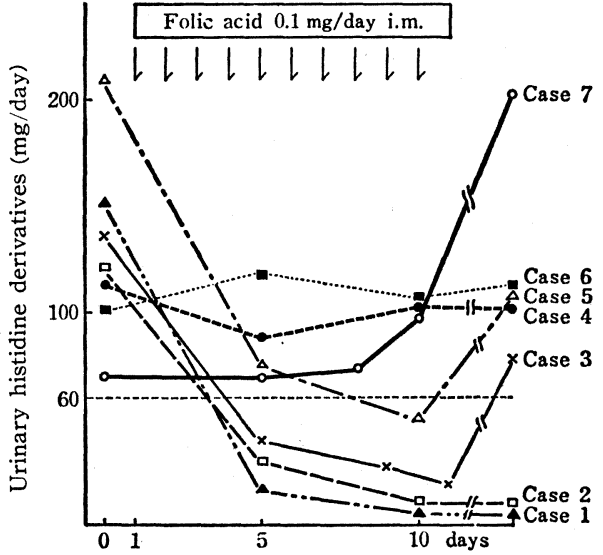

FIG. 3 Effect of daily minimal doses of folic Acid on urinary histidine derivatives in 7 patients with liver diseases.

Chronic hepatitis : Case 1,2,3,4, and 5. Liver cirrhosis, case 6 and 7 .

hematological findings and liver function. No definite improvement of liver function was observed following the doses of folic acid, while the hematological response of reticulocytosis was observed in all the cases, though the degree of increse of reticulocyte count was not directly related to that of histidine derivatives in amount. These were almost in concordance with the result of Fig. 2. Therefore, it would be naturally anticipated that some other factors such as the lowered activity of liver enzyme would be involved in the increased excretion of the derivatives. For investigating the assumption, THF was administered to Case $3,4,5$, and 7 in which the urinary histidine derivatives were either refractory or transiently responsive to the folic acid therapy. The dose and duration of THF administration were the same as those of folic acid, daily dose of $0.1 \mathrm{mg}$ of THF for ten days. The result is shown in Fig. 5.

The two cases (Case 3 and 5) showed an immediate decrease of the derivatives to a normal range after the dose of THF, and one of the remaining two cases (Case 7), which was refractory to the treatment of folic acid, responded to THF, resulting in a marked decrease of the derivatives, though the pretreatment level with THF was far larger 

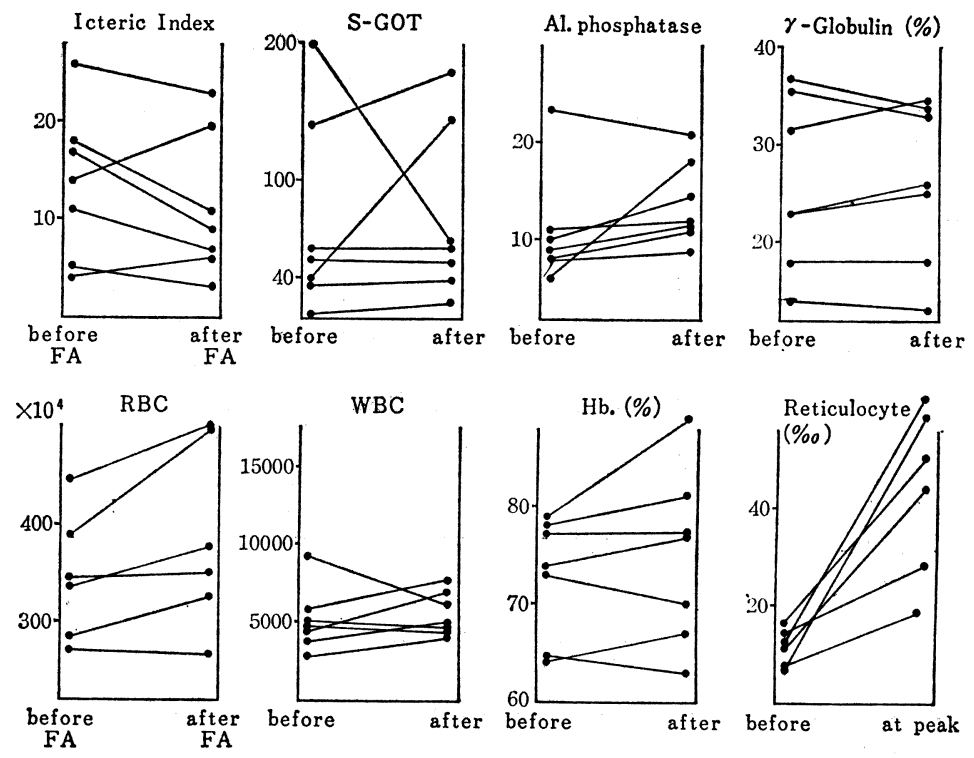

FIG. 4 Liver function tests and hematological findings before and after the treatment with folic acid in 7 patients with liver diseasis.

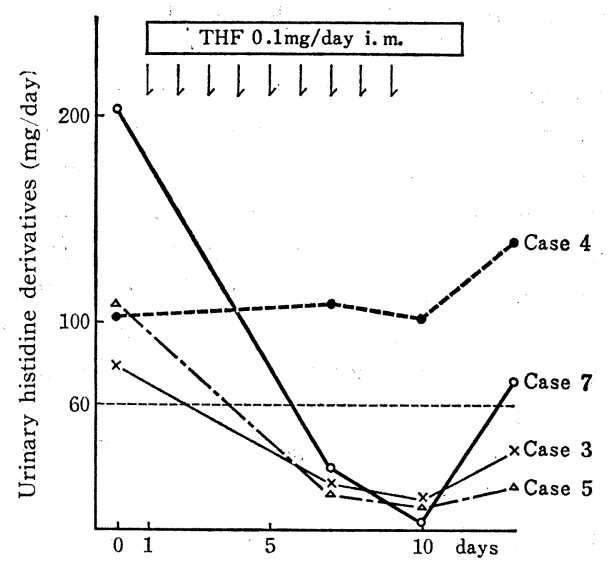

FIG. 5 Effect of daily minimal doses of THF on urinary histidine derivatives in 4 patients with liver diseases.

than that with folic acid.

In case 4 , either folic acid or THF failed to decrease the amount of the derivatives, indicating possibly the lowered activity of FIGLU-transferase.

3. Therapeutic Trials with Vitamin $B_{12}$ and Coenzyme $B_{12}$

Following the folic acid and THF treatment, two cases (Case 4 and 7) were selected for the observation of $t$ effect of DBCC and CN-] on the urinary excretion the derivatives. $\mathrm{CN}-\mathrm{B}_{12}$ n first administered and th DBCC intramuscularly. $\mathrm{T}$ results are shown in Fig.

Case 7 , a male aged ! had a partial gastrectomy : months before admission. $\mathrm{T}$ laboratory findings on adm sion included icteric index 24 , cobalt reaction of $\mathrm{R}_{9}, \mathrm{SGC}$ over 200 units, alkaline phr phatase 7.5 units, and chol, terol of $74 \mathrm{mg} / 100 \mathrm{ml}$. T examination of blood disclos $55 \%$ hemoglobin, $3.1 \times 10^{6} \mathrm{r}$ blood cell, 4,000 white blo cell. From these findings a liver biopsy, the diagnosis liver cirrhosis was establish The urinary excretion of histidine derivativ which had been slightly increased during fo acid, administration was markedly increas before THF administration which resulted improvement of abnormal histidine derivativ When the histidine derivatives were increas after THF administration, $\mathrm{CN}-\mathrm{B}_{12}$ and $\mathrm{DBC}$ were given but failed to improve the excr sive histidine derivatives.

Case 4, a male aged 64, had the subto1 gastrectomy because of carcinoma of $t$ stomach about eight years before admissi and received a large quantity of blood trar fusion. The liver function tests and the liv biopsy indicated chronic active hepatitis, a the hematological examination showed hyp chromic, macrocytic anemia with megaloblas 1 bone marrow. This patient made no respon to the pretreatment of folic acid and $\mathrm{TH}$ and the excretion of the derivatives befo $\mathrm{CN}-\mathrm{B}_{12}$ administration in which urocanic ac was predominant, was much larger than th in the previous doses of folic acid and TH The patient was given intramuscularly fir $0.1 \mu \mathrm{g} /$ day of $\mathrm{CN}-\mathrm{B}_{12}$ for successive five das and next, in the same way, of DBCC. $T$, days after the initiation of $\mathrm{CN}-\mathrm{B}_{12}$, the urina 

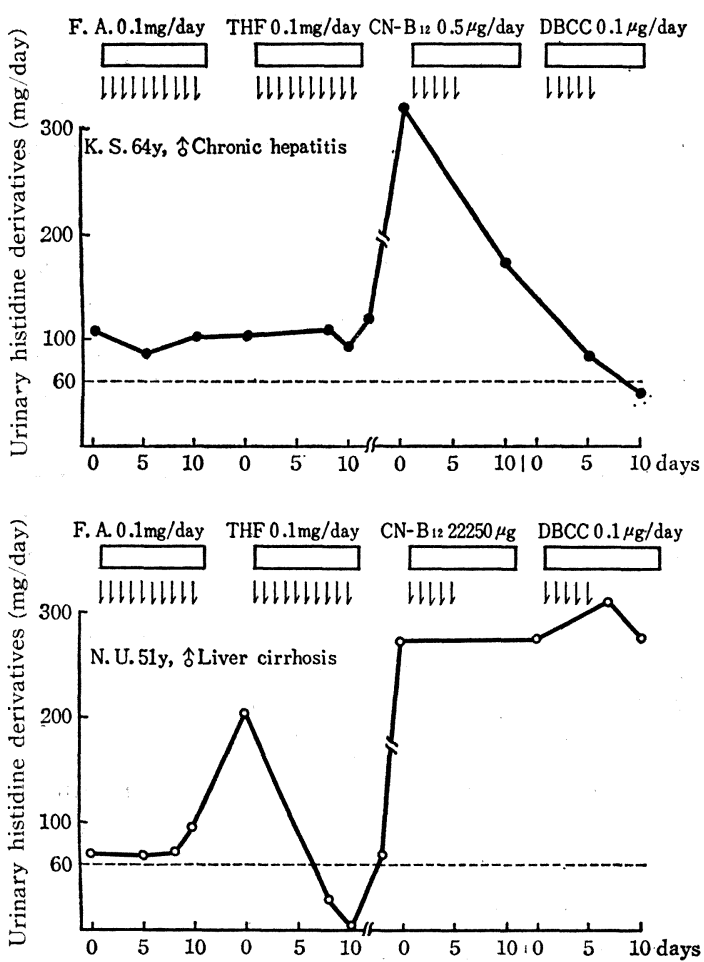

FIG. 6 Effect of folic acid and vitamin $B_{12}$ derivatives on urinary excretion of histidine derivatives in two patients with liver disease.

The upper figure was was of Case 4, and the lower, of Case 7.

derivatives was markedly decreased but still remained above the normal range. However, after DBCC, the derivatives returned to the normal range.

In brief, in case 4 it was observed that folic acid and THF were ineffective but either $\mathrm{CN}-\mathrm{B}_{12}$ or $\mathrm{DBCC}$, or both were effective for decreasing the excessive excretion of the derivatives. Therefore, it was reasonably speculated that lowered activity of FIGLU. transferase probably existed in this case. To the contrary, folic acid, $\mathrm{CN}-\mathrm{B}_{12}$ and DBCC were not effective in case 7 . Only THF resulted in a decrease of the excessive histidine derivatives.

\section{Discussion}

The present study revealed that, using histidine derivatives as an index for estimating the degree of folic acid deficiency, there existed folic acid deficiency among patients with liver diseases more frequently that previously noted. A considerable high percentage of patients with acute and chronic hepatitis, liver cirrho sis, and liver cancer was observed to excrete an abnormal amount of the derivatives in urine, but there was no significant difference in urinary excretion of the derivatives among each of various types of liver diseases.

Abnormal amount of histidine derivatives have been found repeatedly in the urine of the majority of patients with liver cirrhosis $(5,19,24,33)$ and other liver diseases as well as thyrotoxicosis, hemolytic anemia, Hodgkin's disease, congestive heart failure and intestinal malabsorption (7), and various neoplastic diseases $(4,18,20)$. Merritt et al. have reported excessive excretion of FIGLU and excessive or normal excretion of urocanic acid after a small dose of histidine in seven cases of miscellaneous liver diseases (24).

The mechanism of an increased histidine derivatives in the liver diseases may be due to the primary or secondary folic acid deficiency and due to inactivation of FIGLUtransferase. The primary deficiency implies that the total body store of folic acid is diminished to such an extent as to give rise to certain effects which are correctable by small (50-100 $\mu \mathrm{g}$ daily) doses of folic acid $(22,36)$. The sedondary deficiency defines as a state in which the body store of folic acid is believed to be normal but the same untoward effects arise, because the folic acid cannot be utilized and these effects are only corrected, if at all, by massive doses of folic acid. Such a secondary deficiency is observed in patients treated with folic acid antagonists because folic acid metabolism is blocked, and in pernicious anemia because vitamin $B_{12}$ is apparently needed as a coenzyme in the conversion of the stored folic acid to an active form $(17,22)$ and in liver diseases because the activity of liver enzymes is decreased. Knowles et al. and Carter et al. have reported that secondary deficiency of folic acid is common in liver diseases $(5,19)$. 
Reports of the value of small doses of folic acid in the differentiation of the causes of megaloblastic anemia have appeared $(22,36)$. Although the methods and dosage schedules employed have varied so that a standard procedure has not been established. The dose range of the therapeutic trial used in the present study was designed to be of minimal order, so as not to elicit a nonspecific response or mass action effect by the nonspecific vitamin. Therefore, the dose of folic acid and THF was decided in the order of $0.1 \mathrm{mg} /$ day for 10 days, fundamentally.

The results of the present study demonstrated that four out of seven cases responded to the folic acid therapy, and a significant decrease of the urinary histidine derivatives was observed. The results suggest the existence of the primary folic acid deficiency in these cases.

It has been reported that low levels of serum folic acid always appeared with primary deficiency $(11,35)$. In pernicious anemia the levels of folic acid has been found normal or high, and inversely proportional to the serum vitamin $\mathrm{B}_{12}$ (35). Herbert and Zalusky have reported that the low levels of serum folic acid were detected in 65 of 70 alcoholic cirrhosis (12) and Knowles et al. also have reported that the same observations were found in four of five cases (19). According to Knowles' interpretation, a dietary deficiency of folic acid would be more common in alcoholic patients and this might account for lower levels of serum folic acid and higher levels of excretion of FIGLU. Although there are fewer patients with alcoholic cirrhosis in Japan than abroad, the simple folic acid deficiency exists in four out of seven patients studied.

In addition, it has been postulated that impaired storage or decreased hepatic activity for folic acid might result in depletion of the vitamin in chronic liver disease (19). However, it was found in the present study that there was no direct correlation between the severity of urinary excretion of the derivatives and the degree of liver impairment. It was apparent from the present study that marked impairment of liver function does not necessarily result in a large amount of histidine derivative excretion. In brief, inadequate dietary intake of folate seems to be of great importance in the development of folic acid deficiency in liver disease.

In the present study, three of four cases treated with THF responded. Two cases of these three responded to both folic acid and THF, and the remaining one did to THF only. The fourth case was refractory to both folic acid and THF. In four patients treated with THF, two had a simple deficiency of folic acid, and two had the secondary deficiency of the vitamin. In these two cases, there may exist the inactivation of dihydrofolate reductase, methyltetrahydrofolate transferase or FIGLU-transferase. Folic acid is metabolically active only in the forms of THF and several liver enzymes are necessary for the conversion. If the activities of the enzymes are lowered in liver diseases, the amount of THF pool decreases and consequently, histidine derivatives accumulate in urine.

On the other hand, the FIGLU-transferase is required for the conversion of FIGLU to glutamic acid. The inactivation of FIGLUtransferase causes an ac cumulation of FIGLU even in the presence of THF.

In the present study using four compounds, Case 7 suggests an inactivation of dihydrofolate reductase, but case 4 responded only to vitamin $\mathrm{B}_{12}$. Although an increased urinary excretion of FIGLU in vitamin $\mathrm{B}_{12}$-deficient human beings $(6,8)$ and in experimental animals $(4$, 29,30 ) has been reported, the mechanism of this process has not been clarified. Herbert and Zalusky demonstrated that in $\mathrm{B}_{12}$ deficiency, $N^{5}$-methyl THF accumulates after the administration of THF (12). However, Baker et al. suggested that increased FIGLU excretion in vitamin $B_{12}$ deficiency might be due to a defect in the synthesis or activation of FIGLU-transferase (1). In the recent report of Vitale et al., decreases in the activity of FIGLU-transferase was demonstrated for the first time in the liver of vitamin $\mathrm{B}_{12}$-deficient rats (34).

If $N^{5}$-methyl THF accumulates and THF 
decreases invitamin $B_{12}$ deficiency, there may be insufficient THF available for the metabolism of FIGLU to glutamic acid. On the other hand, several explanations are assumed for the decreased activity of FIGLU-transferase in vitamin $\mathrm{B}_{12}$ deficiency: (a) vitamin $\mathrm{B}_{12}$ may be required for the synthesis or activation of the enzyme, $(b)$ the synthesis of the enzyme may be depressed when the level of THF is low.

The Case 4 which responded to vitamin $\mathrm{B}_{12}$ and not to THF demonstrated the existence of the lowered activity of FIGLU-transferase indirectly, though the activity of the enzyme itself was not measured.

\section{ACKNOWLEDGMENT}

Grateful acknowledgement is made to Professor G. Wakisaka, Director, The First Division, Department of Internal Medicine, Kyoto University, for his constant interest and guidance; to Professor H. Uchino, Director of Internal Medicine, Research Institute for Nuclear Medicine and Biology, Hiroshima University, for his direction; to Dr. H. Sotobayashi, Assistant, The First Division, Department of Internal Medicine, Faculty of Medicine, Kyoto University, for his directions and to Miss Sakon for her technical assistance. This paper was partly presented at the Japan Vitamin Society 20th Meeting, April, 1968.

\section{REFERENCES}

1. Baker, H., Frank, O., Gellene, R. A., and Levy, C. M., Biol. Med., 117, 492 (1964).

2. Bennett, M. C., and Chanarin, I., Lancet, ii, 1095 (1961).

3. Bennett, M. C., and Chanrin, I., Nature, 196, 271 (1962).

4. Brown, D. D., Silva, O. L., Gardiner, R. C., and Silverman, M., J. Biol. Chem., 235, 2058 (1960).

5. Carter, F. C., Heller, P., Schaffner, G., and Korn, R. J., Arch. Int. Med., 108, 41 (1961).

6. Chanarin, I., Brit. J. Haemat., 9, 141 (1963).

7. Chanarin, I., and Bennett, M. C., Brit. Med. J., 1, 27 (1962).

8. Chanavin, I., Bennett, M. C., and Berry, V., J. Clin. Path., 15, 269 (1962).

9. Chanarin, I., Mollin, D. L., and Anderson, B. B., Proc. Roy. Soc. Med., 51, 269 (1962).

10. Daft, F.S., in Liver Function, edited by R.W.
Brauer, Washington, D. C., Amer. Inst. Biol. Sci., p. $360,368,370$ (1958).

11. Herbert, V., Fisher, R., and Koontz, B. J., J. Clin. Invest., 40, 81 (1961).

12. Herbert, V., and Zalusky, R., J. Clin. Invest., 41, 1263 (1962).

13. Ishihara, S., In press.

14. Jarrold, T., and Vilter, R. W., J. Clin. Invest., 28, 286 (1949).

15. Klipstein, F. A., and Lindenbaum, J., Blood., 25, 443 (1965).

16. Knowles, J. P., Lancet, ii, 1149 (1961).

17. Knowles, J. P., and Prankerd, T. A. J., Clin. Sci., 22, 233 (1962).

18. Knowles, J.P., Prankerd, T. A.J ., and Westall, R. G., Lancet., ii, 347 (1960).

19. Knowles, J.P., Shaldon, S., and Fleming A., Clin. Sci., 24, 39 (1963).

20. Kohn, J., Mollin, D. L., and Rosenbach, L. M., J. Clin. Path., 14, 345 (1961).

21. Luhby, A. L., Cooperman, J.M., and Teller, D. N., Am. J. Clin. Nutr. 7, 393 (1958).

22. Marshall, R. A., and Jandl, J.H., Arch. Int. Med., 105, 352 (1960).

23. Merritt, A. D., Ruchnagel, D. L., Gardiner, R. C., and Silverman, M., Clin. Res., 8, 213 (1960).

24. Merritt, A. D., Ruchnagel, D. L., Silverman, M., and Gardiner, R. C., J. Clin. Invest., 41, 1472 (1962).

25. Miller, A., and Waelsch, H., Arch. Biochem. Biophys., 63, 263 (1956).

26. Miller, A., and Waelsch, H., J. Biol. Chem., 228, 397 (1957).

27. Movitt, E. R., Blood, 5. 468 (1950).

28. Silerman, M., Gardiner, R., and Bakerman, H., J. Biol. Chem., 194, 185 (1952).

29. Silverman, M., and Pitney, J., J. Biol. Chem., 233. 1179 (1958).

30. Stokstad, E. L. R., Webb, R. E., and Shah, E., J. Nutrition., 88, 225 (1966).

31. Tabor, H., and Rabinowitz, J., J. Am. Chem. Soc., 78, 5705 (1956).

32. Tabor, H., Silverman, M., Mehler, A. H., Daft, F.S., and Bauer, H., J. Am. Chem. Soc., 75, 756 (1953).

33. Villamil. A., and McCracken, B. H., Brit. Med. $J ., 1,717$ (1963).

34. Vitale, J. J., and Hegsted, D. M., Am. J. Clin. Nutr., 20, 311 (1967).

35. Waters, A. H., and Mollin, D. L., J. Clin. Path., 14, 335 (1961).

36. Zalusky, R., and Herbert, V., New Eng. J. Med., 265, 1033 (1961). 